\title{
NONLINEAR PREDICTIVE ATTITUDE CONTROL OF SPACECRAFT UNDER EXTERNAL DISTURBANCE
}

\author{
Hyunsam Myung, Hyochoong Bang ${ }^{*}$, Choongsuk Oh, Min-Jea Tahk \\ Dept. of Aerospace Engineering, \\ Korea Advanced Institute of Science and Technology (KAIST)
}

\begin{abstract}
Predictive control technique is applied to the three-axis attitude control of spacecraft. The principal idea of predictive control is to construct a priori reference trajectory and build control command so that the actual system follows the reference trajectory. In the case of this study, the controlled variables are the quaternion attitude parameters and angular rates of spacecraft body axes. The key objective of this paper is to design a predictive controller for the three-axis attitude control under external disturbances. Designing a predictive control law including disturbances and estimation of the disturbance are discussed. Conventional predictive controller design approach has been partially modified in conjunction with disturbance identification strategy. The results of this paper, attitude control simulation results, are presented to prove this research. Copyright $(2002$ IFAC
\end{abstract}

Keywords: Spacecraft autonomy, predictive control, attitude control, quaternion feedback, disturbance rejection.

\section{INTRODUCTION}

Satellite attitude dynamics are basically governed by nonlinear equations due to inherent gyroscopic coupling effect which arises from three-axis rotational motion. The Euler equation describes dynamic equilibrium between system angular momentum vector and applied torque inputs. The nonlinearity of governing equations require nonlinear control law in general even though, in some regions of interest, linearized version of governing equations may be used to design linear control laws. There has been significant effort in nonlinear control laws design for the spacecraft attitude maneuver, in particular, large angle attitude maneuvers.

As a nonlinear control design approach, the so-called predictive control uses future reference trajectories to design a control law based upon the reference trajectories and current states of the given system. The nonlinear predictive control law has been recently applied to control of aerospace systems such as aircraft, missiles, and spacecraft(Lu, 1994). In particular, the spacecraft large angle maneuver control law as well as filtering problem have been investigated by Crassidis et al. (1997). The central feature of the predictive control is to build a control input in such a manner that the trajectory of actual system follows that of the reference system.

Crassidis et al. (1997) applied the predictive control technique to three-axis spacecraft attitude control. Both quaternion attitude parameters and angular velocity components of spacecraft body axes are taken as control variables. Also, predictive filtering has been investigated as a dual problem to the controller design case. In this case, system modelling error was taken as external disturbance input which is estimated in the sense of minimum estimation error. The cost function is therefore selected in the form of error covariance for the filtering problem.

In majority of previous studies for predictive control, external disturbance has not been considered in the system dynamics. Only control input is used to describe system dynamics, which is not practically true. In other words, external disturbance and control input act together to excite system dynamics. Spacecrafts in general mission are easily subject to various environmental disturbance sources such as solar pressure, gravitational disturbance, earth magnetic field effect, and other internal sources.

In this paper a predictive control design approach is proposed for the application to the spacecraft nonlinear attitude maneuvers. This study could be considered as an extension of a previous study by Crassidis (1997) by explicitly including external disturbance input in the system dynamics. Then the control law is designed in such a manner to handle the disturbance directly. The disturbance is also estimated on the condition that the tracking error energy is kept at minimum by the designed control law in conjunction with the disturbance input. 


\section{GOVERNING EQUATIONS OF MOTION AND KINEMATICS}

Before the control law design is discussed, the governing equations of motion for spacecraft attitude motion are presented first. The Euler equation produces equations of motion for rotational dynamics as

$$
\dot{\boldsymbol{H}}=-\omega \times \boldsymbol{H}+\boldsymbol{u}_{\text {ext }}
$$

where $\boldsymbol{H}$ represents system angular momentum vector, $\boldsymbol{\omega}$ is angular velocity vector, and $\boldsymbol{u}_{\boldsymbol{e x t}}$ denotes external torque input vector which includes both control and disturbance torque inputs. If spacecraft mass moment of inertia is denoted as $J$, then the angular momentum $(\boldsymbol{H})$ vector can be written as $J \omega$. So that the equation of motion in Eq. (1) becomes

$$
\dot{\omega}=-J^{-1} \omega \times\left(\begin{array}{ll}
J & \omega
\end{array}\right)+J^{-1} u_{e x t}
$$

In addition to rotational dynamics, attitude kinematics is needed to describe spacecraft motion completely. Quaternion is a popular attitude representation method without singularity problem. The quaternion elements are defined as (Sidi, 1997)

$$
\begin{gathered}
\boldsymbol{q}=\left[\begin{array}{l}
\boldsymbol{q}_{13} \\
q_{4}
\end{array}\right], \boldsymbol{q}_{13} \equiv\left[\begin{array}{l}
q_{1} \\
q_{2} \\
q_{3}
\end{array}\right] \equiv \hat{\boldsymbol{n}} \sin \left(\frac{\theta}{2}\right) \\
q_{4} \equiv \cos \left(\frac{\theta}{2}\right)
\end{gathered}
$$

where $\hat{\boldsymbol{n}}$ is the vector of Euler's principal axis and $\theta$ represents Euler's principal angle in Euler's principal axis rotation theorem. Obviously, quaternions satisfy the following constraint equation

$$
q_{1}^{2}+q_{2}^{2}+q_{3}^{2}+q_{4}^{2}=1
$$

The time rate of change of quaternions can be related to the spacecraft body axis angular velocity components (Sidi, 1997)

$$
\dot{\boldsymbol{q}}=\frac{1}{2} \Omega(\omega) \boldsymbol{q}=\frac{1}{2} \Xi(\boldsymbol{q}) \omega
$$

where each symbol is given by

$$
\begin{gathered}
\Omega(\omega) \equiv\left[\begin{array}{ccc}
-[\omega \times] & \vdots & \omega \\
\cdots & & \cdots \\
-\omega^{T} & \vdots & 0
\end{array}\right] \\
\Xi(\boldsymbol{q}) \equiv\left[\begin{array}{c}
q_{4} I_{4 \times 4}+\left[q_{13} \times\right] \\
\cdots \ldots \ldots \ldots \\
-\boldsymbol{q}_{13}^{T}
\end{array}\right]
\end{gathered}
$$

and the following notation was employed.

$$
[a \times]=\left[\begin{array}{ccc}
0 & -a_{3} & a_{2} \\
a_{3} & 0 & -a_{1} \\
-a_{2} & a_{1} & 0
\end{array}\right]
$$

Difference $\left(\boldsymbol{q}^{\prime}\right)$ between two quaternion elements $(\boldsymbol{q}$, $\boldsymbol{q}^{\prime}$ ) can be represented as (Sidi, 1997)

$$
\boldsymbol{q}^{\prime \prime}=\left[\begin{array}{cccc}
q_{4}^{\prime} & q_{3}^{\prime} & -q_{2}^{\prime} & q_{1}^{\prime} \\
-q_{3}^{\prime} & q_{4}^{\prime} & q_{1}^{\prime} & q_{2}^{\prime} \\
q_{2}^{\prime} & -q_{1}^{\prime} & q_{4}^{\prime} & q_{3}^{\prime} \\
-q_{1}^{\prime} & -q_{2}^{\prime} & -q_{3}^{\prime} & q_{4}^{\prime}
\end{array}\right]\left[\begin{array}{c}
q_{1} \\
q_{2} \\
q_{3} \\
q_{4}
\end{array}\right]
$$

The above relationship is used to determine error quaternions between the desired and the actual trajectory of systems. If the errors are to be so small then it can be seen that $\boldsymbol{q}_{13}^{\prime \prime}$ approaches zero while $q_{4}^{\prime \prime}$ approaches unity.

\section{PREDICTIVE CONTROL DESIGN}

\subsection{Predictive Control Theory}

Let us consider a nonlinear system dynamics represented in the form

$$
\begin{aligned}
\dot{\boldsymbol{x}}(t) & =f(\boldsymbol{x}(t))+G(\boldsymbol{x}(t)) \boldsymbol{u}(t)+D(\boldsymbol{x}(t)) \boldsymbol{d}(t) \\
\boldsymbol{y}(t) & =c(\boldsymbol{x}(t))
\end{aligned}
$$

The above system description is directly associated with spacecraft attitude dynamics presented in the previous section. The notations are $\boldsymbol{x}(t) \in R^{n}$ for state vector, $\boldsymbol{u}(t) \in R^{p}$ for control input vector, $\boldsymbol{d}(t) \in R^{q}$ for external disturbance input, and $\boldsymbol{y}(t) \in R^{m}$ for system output vector. The output function given in Eq. (8b) is designed to follow a reference trajectory by the control command to be designed. Also, the disturbance influence function $D(x(t))$ is tentatively assumed to be identified exactly. This rather ideal assumption still does not mislead the original objective of this study.

As the first step of predictive control design, the output function in Eq. (8b) at time $t+\Delta t$ is defined in terms of other variables at time $t$. Taylor series expansion is applied to derive such relationships. By using Lie derivative notations, therefore the following relationship is resulted.

$$
\begin{aligned}
\boldsymbol{y}(t+\Delta t)= & \boldsymbol{y}(t)+\boldsymbol{z}(\boldsymbol{x}(t), \Delta t)+\Lambda(\Delta t) S_{G}(\boldsymbol{x}(t)) \boldsymbol{u}(t) \\
& +\Lambda(\Delta t) S_{D}(\boldsymbol{x}(t)) \boldsymbol{d}(t)
\end{aligned}
$$

where $z(x(t), \Delta t) \in R^{m}$ is also represented in the form of Lie derivative such as (Crassidis et al., 1997)

$$
z_{i}(\boldsymbol{x}(t), \Delta t)=\sum_{k=1}^{p_{i}} \frac{\Delta t^{k}}{k !} L_{f}^{k}\left(c_{i}\right)
$$


Note that $p_{i}, \quad i=1,2, \ldots, m$ is the order of derivative of $c_{i}(\boldsymbol{x}(t))$ for which the control input appears explicitly for the first time. $L_{f}^{k}\left(c_{i}\right)$ represents $k$-th Lie derivative satisfying ( $\mathrm{Lu}, 1994$ )

$$
\begin{gathered}
L_{f}^{k}\left(c_{i}\right) \equiv c_{i} \quad \text { for } \quad k=0 \\
L_{f}^{k}\left(c_{i}\right) \equiv \frac{\partial L_{f}^{k-1}\left(c_{i}\right)}{\partial \boldsymbol{x}} \boldsymbol{f} \quad \text { for } \quad k \geq 1
\end{gathered}
$$

where $\Lambda(\Delta t) \in R^{m \times m}$ is a diagonal matrix with diagonal components given as (Lu, 1994)

$$
\lambda_{i i}=\frac{\Delta t^{p_{i}}}{p_{i} !} \quad i=1,2, \ldots, m
$$

In addition both $S_{G}(x(t)) \in R^{m \times p}$ and $S_{D}(x(t)) \in R^{m \times q}$ can be defined in terms of matrices which consist of the following row elements (Crassidis et al., 1997) .

$$
\begin{array}{ll}
\left(s_{G}\right)_{i}=\left\{L_{G_{i}}\left[L_{f}^{p_{f}-1}\left(c_{i}\right)\right], \ldots, L_{G_{p}}\left[L_{f}^{p_{i}-1}\left(c_{i}\right)\right]\right\}, & i=1,2, \ldots, m \\
\left(s_{D}\right)_{i}=\left\{L_{D_{i}}\left[L_{f}^{p_{f}-1}\left(c_{i}\right)\right], \ldots, L_{D_{q}}\left[L_{f}^{p_{i}-1}\left(c_{i}\right)\right]\right\}, & i=1,2, \ldots, m
\end{array}
$$

for which the Lie derivatives satisfy

$$
\begin{array}{rlrl}
L_{G_{j}}\left[L_{f}^{p_{i}-1}\left(c_{i}\right)\right] & \equiv \frac{\partial L_{f}^{p_{i}-1}\left(c_{i}\right)}{\partial \boldsymbol{x}} G_{j} & j=1,2, \ldots, p \\
L_{D_{j}}\left[L_{f}^{p_{i}-1}\left(c_{i}\right)\right] \equiv \frac{\partial L_{f}^{p_{i}-1}\left(c_{i}\right)}{\partial \boldsymbol{x}} D_{j} & j=1,2, \ldots, q
\end{array}
$$

Based upon derivations made here, predictive controller design is followed in the next.

\subsection{Predictive Controller Design}

The derivations resulted in the previous section are used to build a nonlinear predictive control law. As early mentioned, the external disturbance input $(\boldsymbol{d}(t))$ is directly accommodated in the controller design as it appears explicitly in the system dynamics.

First, a cost function which consist of tracking error energy, control input $(\boldsymbol{u}(t))$, and external disturbance $(\boldsymbol{d}(t))$ is defined first. The cost function is in the form of a Lyapunov function as follows

$$
\begin{aligned}
J(\boldsymbol{u}(t), \boldsymbol{d}(t))= & \{\tilde{\boldsymbol{y}}(t+\Delta t)-\boldsymbol{y}(t+\Delta t)\}^{T} \\
& \times R\{\tilde{\boldsymbol{y}}(t+\Delta t)-\boldsymbol{y}(t+\Delta t)\} \\
& +\boldsymbol{u}^{T}(t) W \boldsymbol{u}(t)+\boldsymbol{d}^{T}(t) H \boldsymbol{d}(t)
\end{aligned}
$$

where $\tilde{y}(t+\Delta t)$ is the desired output at time $t+\Delta t$ $R \in R^{m \times m}, W \in R^{p \times p}$, and $H \in R^{q \times q}$ represent the weighting matrices on each term including output error, control input and disturbance input. Note that the cost function defined in Eq. (15) is different from that of previous studies by $\mathrm{Lu}$ (1994) and Crassidis et al. (1997). In those cases control input and disturbance were handled separately. In other words, for controller design, only control input $(\boldsymbol{u}(t))$ was considered in the cost function while for the filtering, only modelling error $(\boldsymbol{d}(t))$ was adopted in the cost function.

Now for the given cost function, partial derivatives are taken with respect to $\mathbf{u}(\mathrm{t})$ and $\mathbf{d}(\mathrm{t})$. This operation is intended to find the best set of control input and disturbance simultaneously in the sense of minimum output tracking error. After simple algebra starting from

$$
\frac{\partial J}{\partial \mathbf{u}}=0, \quad \frac{\partial J}{\partial \mathbf{d}}=0
$$

it can be shown that

$$
\left[\begin{array}{l}
\boldsymbol{u}(t) \\
\boldsymbol{d}(t)
\end{array}\right]=\left[\begin{array}{ll}
A_{G G} & B_{G D} \\
B_{D G} & A_{D D}
\end{array}\right]^{-1}\left[\begin{array}{l}
C_{G} \\
C_{D}
\end{array}\right]
$$

where each parameter is defined as

$$
\begin{gathered}
A_{i i}=\left(\Lambda S_{i}\right)^{T} R\left(\Lambda S_{i}\right)+M \\
B_{i j}=\left(\Lambda S_{i}\right)^{T} R\left(\Lambda S_{j}\right) \\
C_{i}=\left(\Lambda S_{i}\right)^{T} R\{\tilde{y}(t+\Delta t)-y(t)-z(x, \Delta t)\}
\end{gathered}
$$

The subscripts are arranged in such a way as

$$
\begin{aligned}
& i=G, j=D, \text { then } M=W \\
& i=D, j=G, \text { then } M=H
\end{aligned}
$$

Equation (16) indicates that the final control law implicitly depends upon the disturbance input due to couplings by system parameters and weighting parameters. Therefore, information on the disturbance input play important role in implementing the control law. In the case of filtering problem by Crassidis and Markley (1997), the disturbance which is considered to be modelling error is estimated on-line.

\subsection{Application into Spacecraft Attitude Control}

In order to apply the control design technique to the spacecraft attitude control problem, first Eqs. (2) and (5) are transformed into a standard nonlinear state equation as defined in Eq. (8). The state vector is then defined as

$$
\boldsymbol{x} \equiv\left[\begin{array}{c}
q \\
\cdots \\
\omega
\end{array}\right]
$$

so that the resultant state space form of governing equations are given by 


$$
\left[\begin{array}{c}
\boldsymbol{q} \\
\cdots \\
\omega
\end{array}\right]=\left[\begin{array}{c}
\frac{1}{2} \Omega(\omega) \boldsymbol{q} \\
\cdots \ldots \ldots \\
-J^{-1} \omega \times(J \omega)
\end{array}\right]+\left[\begin{array}{c}
0 \\
\cdots \\
J^{-1}
\end{array}\right] \boldsymbol{u}(t)+\left[\begin{array}{c}
0 \\
\cdots \\
D
\end{array}\right] \boldsymbol{d}(t)
$$

Since attitude stabilization as well as attitude pointing is a control objective, the output function becomes

$$
y=x
$$

For a given output function, time derivatives over the components of the output function is taken successively until explicit dependency on $u(t)$ is derived. In this special case, the lowest order of derivatives turns out to be

$$
\begin{array}{rlrl}
p_{i} & =2, & i=1, \ldots, 4 \\
p_{i}=1, & i=5, \ldots, 7
\end{array}
$$

By using the order of derivatives, associated matrices can be obtained as (Crassidis et al., 1997)

$$
\begin{gathered}
\Lambda=\frac{1}{2}\left[\begin{array}{ccc}
\Delta t^{2} I_{4 \times 4} & \vdots & 0_{4 \times 3} \\
\ldots & & \ldots \\
0_{3 \times 4} & \vdots & 2 \Delta t I_{3 \times 3}
\end{array}\right] \\
S_{G}(\boldsymbol{x})=\left[\begin{array}{c}
\frac{1}{2} \Xi(\boldsymbol{q}) J^{-1} \\
\ldots \\
J^{-1}
\end{array}\right] S_{D}(\boldsymbol{x})=\left[\begin{array}{c}
\frac{1}{2} \Xi(\boldsymbol{q}) D \\
\ldots \\
D
\end{array}\right]
\end{gathered}
$$

where the $z$ vector defined in Eq. (10) is written as

$$
\boldsymbol{z}=\left[\begin{array}{c}
z_{q} \\
\cdots \\
z_{\omega}
\end{array}\right]
$$

where each parameter can be shown to be

$$
\begin{aligned}
\boldsymbol{z}_{q}= & -\left(\Delta t^{2} / 8\right)\left\{\left(\mathrm{w}^{\prime} \mathrm{w}\right) \boldsymbol{q}+2 \Xi(\boldsymbol{q}) J^{-1}[\mathrm{w} \times] J \mathrm{w}\right\} \\
& +(\Delta t / 2) \Xi(\boldsymbol{q}) \\
\boldsymbol{z}_{\omega}= & -\Delta t J^{-1}[\omega \times] J \omega
\end{aligned}
$$

\section{SIMULATION STUDY}

In order to validate the designed control law, a sample simulation is conducted. The total simulation time is set to 200 seconds. The reference quaternion attitude trajectory is arbitrarily prescribed as Fig. 1.

The mass moment of inertia of spacecraft and weighting functions for the cost function are given by

$$
J=\operatorname{diag}(20,10,15)\left(\mathrm{kg}-\mathrm{m}^{2}\right)
$$

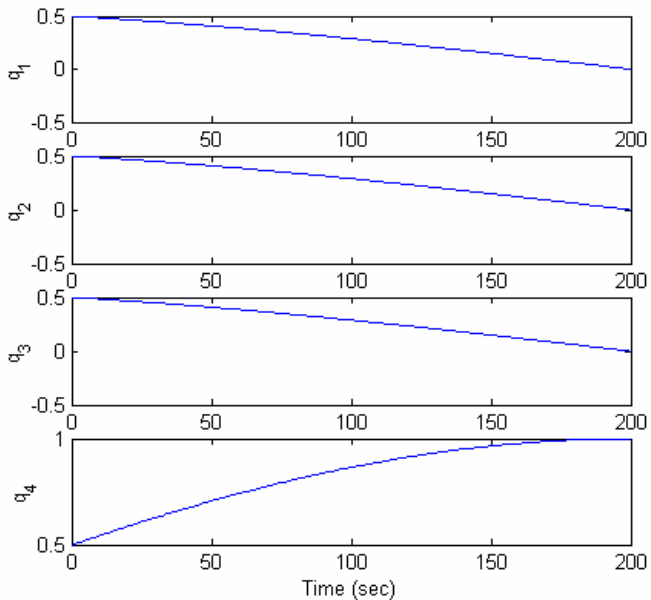

Fig. 1 Reference trajectories of quaternions

and

$$
\begin{gathered}
R=\left[\begin{array}{cc}
r_{q} I_{4 \times 4} & 0_{4 \times 3} \\
0_{3 \times 4} & r_{\omega} I_{3 \times 3}
\end{array}\right], r_{q}=0.1, r_{\omega}=0.2 \\
D=0.2 I_{3 \times 3}, H=0.1 I_{3 \times 3}
\end{gathered}
$$

The initial attitude quaternions and the angular velocity of the actual spacecraft are selected with discrepancy from those of ideal trajectories. The controller performance is then examined whether the actual system follows the reference system with desired tracking performance. It turns out that the simulation results show satisfactory maneuvers in the wide range of the weighting parameter $(W)$ on control input. But the excessive suppression of the control input, or a large value of $W$, results in the poor performance of the attitude tracking. Two different cases with different $W$ are presented herein.

Case I ) $W=0$

Disturbance input $(\mathbf{d}(\mathrm{t}))$ is applied to the system dynamics first, and Eq. (16) is used to determine both control input and disturbance. Simulation results are presented in Figs. 2 and 3. Fig. 2 compares the actual trajectories with those of the reference system. Fig. 3 shows the tracking error trends between the reference and the actual trajectories. As shown, the simulation results indicate a satisfactory tracking maneuver.

Case II) $W=0.1 I_{3 \times 3}$

In this case identical simulation conditions with Case I) are used except for the control weighting parameter which is given by $W=0.1$. Simulation results are plotted in Figs. 4 and 5. Compared to Fig. 2 , sluggish responses in tracking error are observed. This is attributed to the increase in weighting parameter in control input. The increased weighting parameter tends to limit the magnitude of the control command. This value, however, is large enough to be equal to the weighting parameter $(H)$ in the disturbance. 


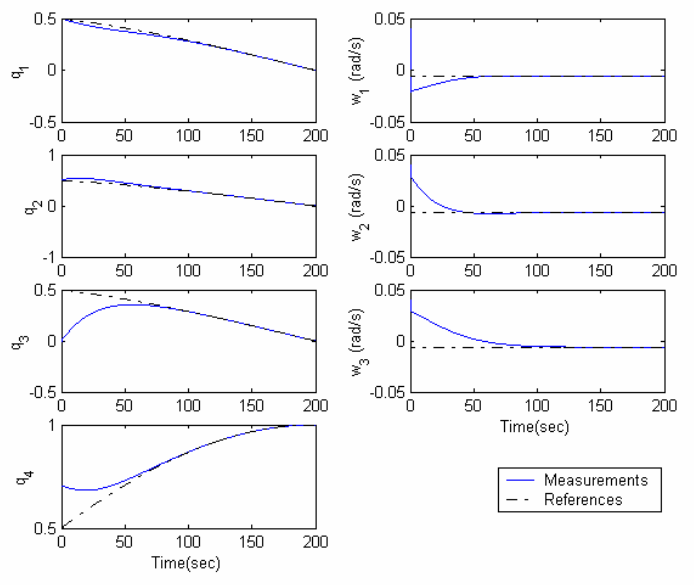

Fig. 2 Reference and the actual trajectories with $W=0$
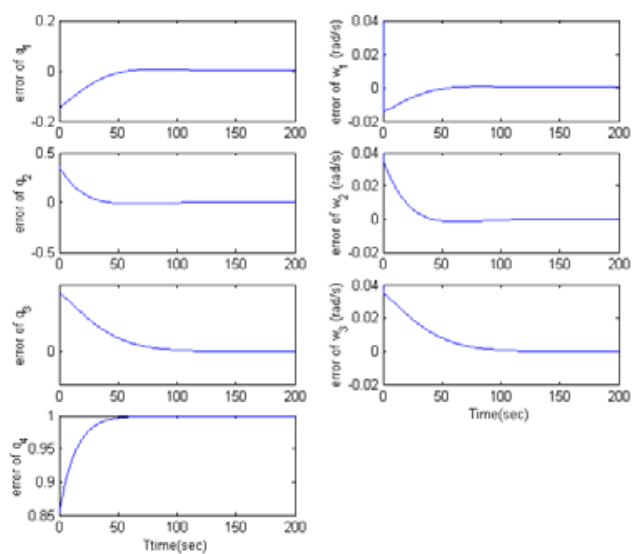

Fig. 3 Tracking error between the reference and the actual trajectories $(W=0)$
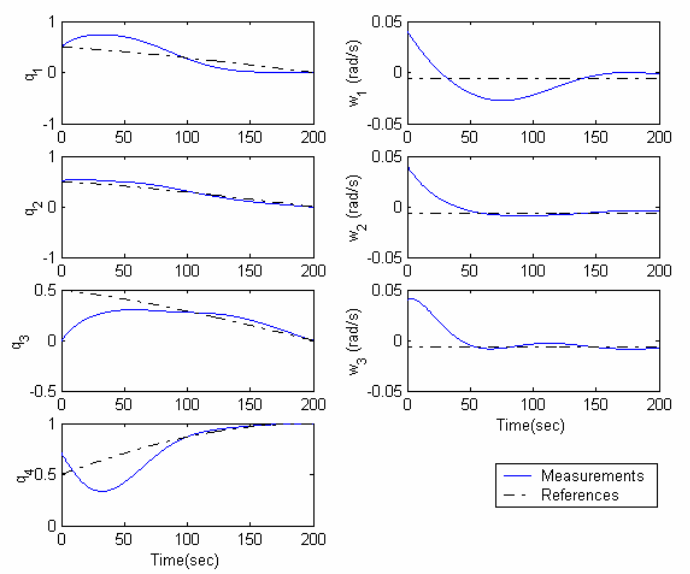

Fig. 4 Reference and the actual trajectories with $W=0.1 I_{3 \times 3}$
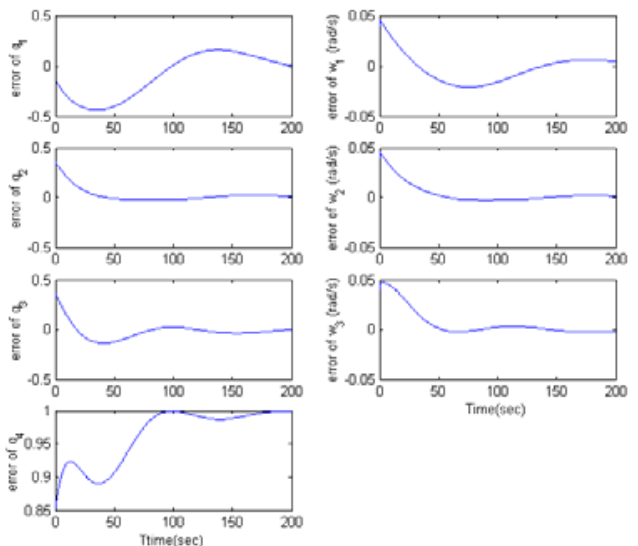

Fig. 5 Tracking error between reference and actual trajectories $\left(W=0.1 I_{3 \times 3}\right)$

The sensitivity of the control command with respect to the weighting parameter is legal since a larger control weighting parameter results in smaller control input and larger disturbance estimation. From the fact, a delicate relationship of the estimated disturbance and the real disturbance should be investigated in the future study.

\section{CONCLUSION}

Predictive control approach has been applied to spacecraft nonlinear attitude maneuvers under the presence of external disturbance. The control input is obtained implicitly in terms of disturbance input. The disturbance input is also derived in a manner to minimize tracking error between reference and actual systems. Simulation results validate the proposed control design technique. The resultant tracking performance is acceptable even if too higher weighting on control input may result in degradation of controlled performance of closed-loop system. Refinement of The relationship of the estimated disturbance and the real disturbance needs in the further study.

\section{REFERENCES}

Crassidis J.L., et al.(1997), "Nonlinear Predictive Control of Spacecraft", Journal of Guidance, Control, and Dynamics, 20, pp. 1096-1103.

Crassidis, J.L., and Markley F.L.(1997), "Predictive Filtering of Nonlinear Systems", Journal of Guidance, Control, and Dynamics, 20, pp. 556572.

Lu, P.(1994), "Nonlinear Predictive Controllers for Continuous Systems", Journal of Guidance, Control, and Dynamics, 17, pp. 553-560.

Sidi, M.J.(1997), Spacecraft Dynamics and Control, New York, the Press Syndicate of the University of Cambridge. 